\title{
LUMINOSITY AND ASTROMETRY OF COMETS: A REVIEW
}

Elizabeth Roemer

Visual Brightness

By far the greatest number of observations of the brightness of comets, and the only ones that cover a long enough time span for investigation of secular effects, have been made by visual methods, mostly with small instruments. Such observations record the contribution of a large part of the coma, and possibly some light from the tail, at wavelengths to which the eye is sensitive. Three distinct observational techniques have been defined:

1) Comparison of similar-appearing extrafocal images of comet and comparison stars for equal apparent brightness. (Most observations have been made by this method.) The technique has been described by Bobrovnikoff (1941a, 1941b).

2) Comparison of the in-focus image of the comet with extrafocal images of comparison stars for equal apparent brightness. This method has been described by Sidgwick (1955).

3) A method used extensively by Max Beyer (1950), in which grossly out-of-focus images of the comet and comparison stars are examined for similarity of extinction against the sky.

Each of these techniques is subject to systematic errors depending upon a variety of factors: the observer and the instrument employed; the observing circumstances, most particularly the brightness of the sky background; and the character of the comet, especially the degree of central condensation. The several techniques, including differences in their susceptibility to 
systematic effects, and physical interpretation of the observational data are considered in some detail by Meisel and Morris (1975).

\section{Photographic Brightness}

Magnitude estimates can also be made from photographic observations. Those derived from photographs taken with small instruments of relatively short $f$ ratio so not differ grossly from visual determinations of "total" brightness. But determinations made from photographs taken with large, relatively long-focus reflectors for astrometric purposes, tend to give much fainter magnitudes. Such photographs are usually taken with the motion accurately compensated, and the images of comets are small, round, and generally quite sharply condensed. These images are often nearly stellar in appearance, so that direct eye comparisons can be made with images of stars on similarly exposed plates of one of the star fields in which photoelectrically calibrated magnitude sequences have been established. The writer has regularly applied a mean correction of 0.3 mag per air mass (blue light) to compensate for differential extinction. Magnitudes derived in such a way are always fainter than those that refer to the brightness of the central condensation observed visually with the same instrument. Typically they are as much as 5-6 magnitudes fainter than visual estimates made with small telescopes using one of the techniques described above.

To determine appropriate exposure times for photographic observations with the large instruments, it has been the practice for some time to compute ephemerides of "nuclear" magnitudes (Roemer 1961). The distinction between "total" $\left(m_{1}\right)$ and "nuclear" $\left(m_{2}\right)$ magnitudes was introduced into the IAU telegram code some years ago, and its general use was recomended by resolution of IAU Commission 20 in 1970 (Trans. IAU XIVB, p. 156, 1971). Actual observations of individual comets will generally fall somewhere 
between "total" and "nuclear" magnitudes. For visual observations there is a well-known and rather pronounced dependence of the observed magnitude on the size of the telescope, the "aperture effect" discussed by Bobrovnikoff (1941a, 1942, 1943). Comets appear systematically fainter the larger the telescope with which they are observed. But it is clear that the $f$ ratio plays a role as well (see, e.g., Morris 1973). "Total" magnitudes fall short of the ideal in that not all of the light from the coma and tail of a well-developed comet is included in observations made visually, even with very small, wide-field instruments. And "nuclear" magnitudes will rarely be free from contamination by light from the inner coma, the amount apparentaly being dependent on the $\underline{f}$ ratio of the telescope and on the characteristics of the comet. Even quite large Schmidt cameras give brightnesses appreciably greater than do the long-focus reflectors. An $f / 4208$-cm reflector gives magnitudes of the order of 1 mag brighter for typical comets than does a $229-\mathrm{cm} f / 9$ instrument.

Interpretation of Nuclear Magnitudes

Not surprisingly it is found as an empirical fact that "nuclear" magnitudes are less sensitive to heliocentric distance than are "total" magnitudes. For a very few comets, direct solution from observed "nuclear" magnitudes over an adequate range of distances has led to an asteroidal-type magnitude law, P/Arend-Rigaux being the outstanding example (Marsden 1974; see also Sekanina 1975). The sensitivity to the heliocentric distance seems to be correlated with the photographic appearance of the comet, in that the more nearly stellar the appearance the closer the brightness behavior is likely to be to a simple reflection law.

Conformity of actual observations to an asteroidal law has been used, supplementing nearly stellar appearance, as a test of the degree of resolution 
of true "nuclear" magnitudes. The investigation by Sekanina (1975) suggests that this may not be a sufficient condition of resolution.

A comet is likely to be of most nearly stellar appearance if observed at large distance from the sun, when it is relatively inactive. But some bright and active comets may be nearly stellar in appearance on shortexposure photographs, particularly if high-contrast photographic emulsions are used. It is, however, a very rare comet that is not imediately recognizable as a comet, whatever the observational circumstances. P/Arend-Rigaux is such an object.

Plates I - VI show the appearance of a number of comets as photographed with long-focus reflectors. Both short- and long-period comets are included, and observations span a considerable range of heliocentric distances. The minor planet (1580) Betulia is shown in Plate VII for comparison.

To the extent that the cometary image is not absolutely stellar in appearance, the "nuclear" magnitudes clearly do not refer exclusively to light reflected from a monolithic nucleus. Dimensions of nuclei calculatea from observations of brightness that include any unresolved contribution from the inner coma will be too large, perhaps considerably so. Even interpreted in a rather uncritical way, the "nuclear" magnitudes determined with the large reflectors have proved that the radii of comet nuclei are in the range from fractions of a kilometer to a few kilometers for typical objects (Roemer 1966). Such dimensions are far below the limit of optical resolution in ordinary circumstances. ${ }^{1}$

IDelseme and Miller (1971) have shown that the brightness profile of continuum light reflected from grains of an icy halo falls off very sharply with distance from the nucleus. Recalling that a radius of $725 \mathrm{~km}$ subtends an angle of $l$ arcsec at a distance of $l$ a.u., It is clear that a significant contribution of light from a grain halo may be included unrecognized in nuclear magnitude estimates when such a halo is present. 


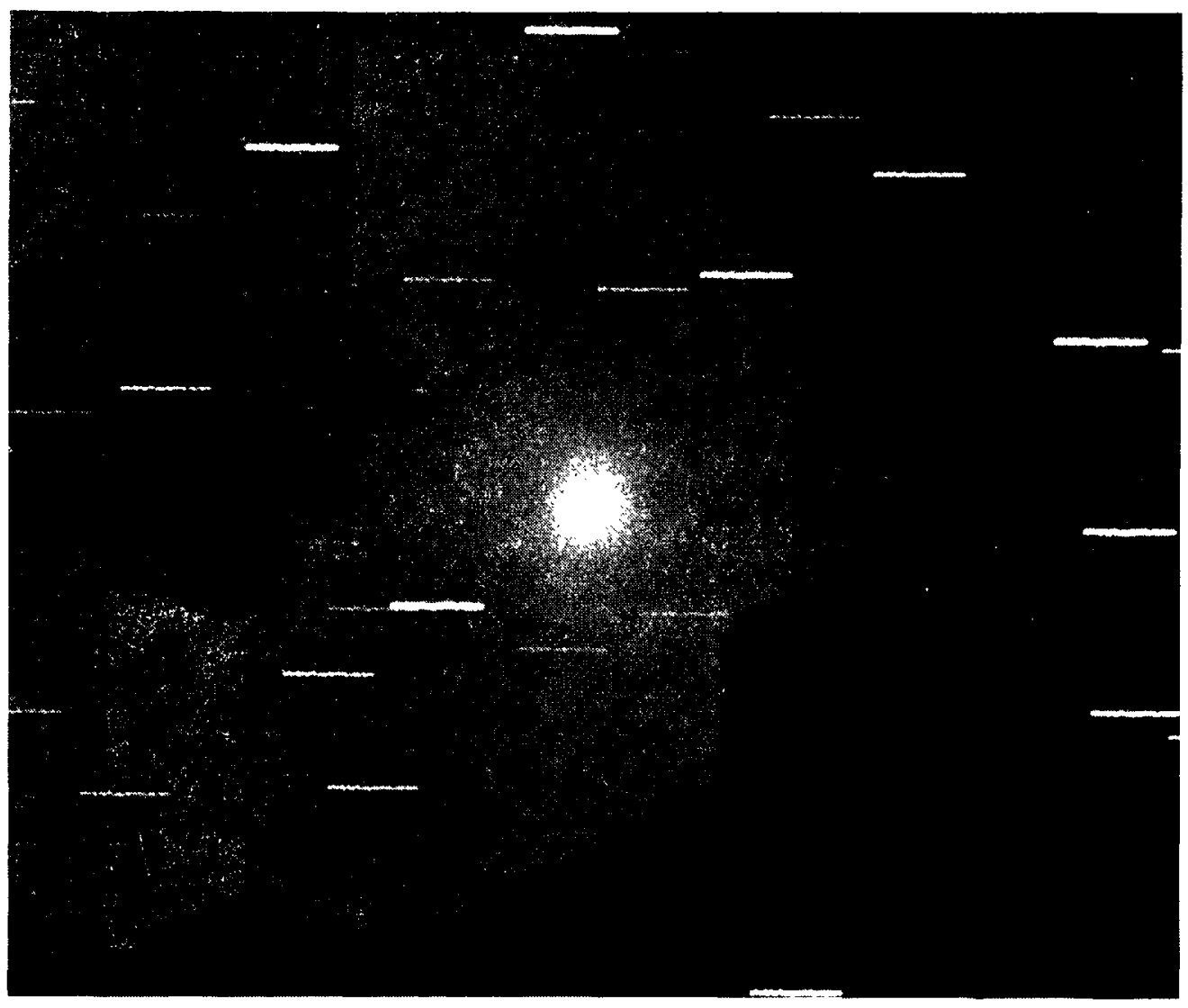

Plate I.

P/Tuttle-Giacobini-Kresák, 1962 V, 1962 Apr. $5 . \Delta=0.27$ a.u., $r=1.15$ a.u. $102-\mathrm{cm} \mathrm{f} / 6.8$ reflector, $30-\mathrm{min}$ exposure on Kodak 103a-0 emulsion. "Very strongly condensed, essentially stellar nucleus in a faint asymetrical coma at least $l^{\prime}$ in diameter." An $m_{2}$ estimate of 16.5 was made from a shorter exposure taken the same night. Note that the quoted descriptions were all made from examination of the original plates. Contrasts are recorded differently in reproductions. Official U.S. Navy Photograph 


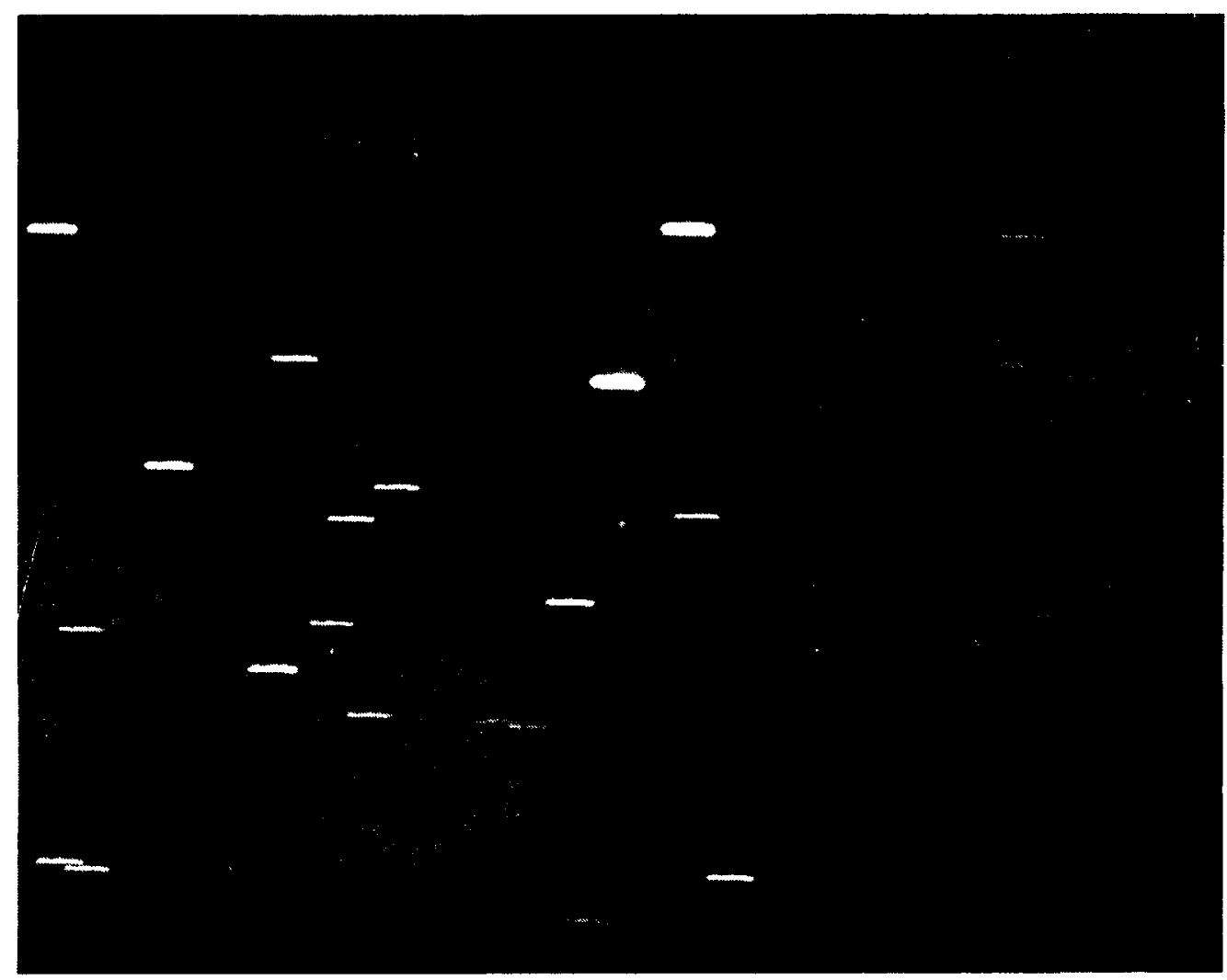

Plate II. P/de Vico-Swift, $1965 \mathrm{VII}, 1965 \mathrm{Aug} .4 . \Delta=0.81$ a.u., $\mathbf{r}=1.64 \mathrm{a} . \mathrm{u}$. $102-\mathrm{cm} \mathrm{f} / 6.8$ reflector, 30-min exposure on Kodak 103a-0 emulsion. "Practically stellar condensation of $\mathrm{m}_{2}$ near 18.7 , with a faint trace of trail . . . WSW". Official U.S. Navy Photograph 


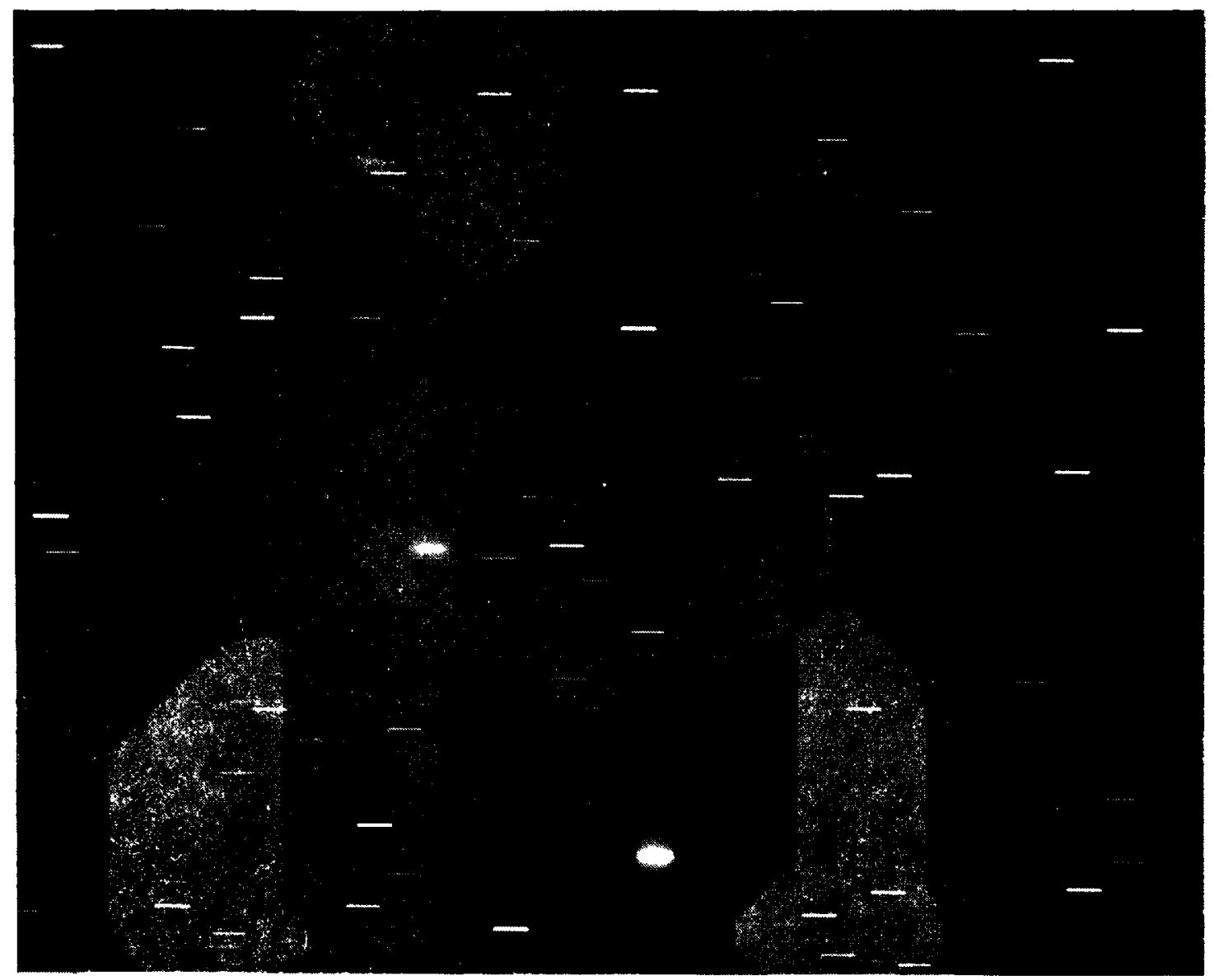

Plate III. P/Tempel 1, 1972 v, 1972 Jan. 1l. $\Delta=1.78$ a.u., $r=2.28$ a.u. 229-cm f/9 reflector, 60-min exposure on Kodak 103a-0 emulsion. "Strong Image; nearly stellar nucleus embedded in a very small, slightly asymmetric coma."

Steward Observatory Photograph 


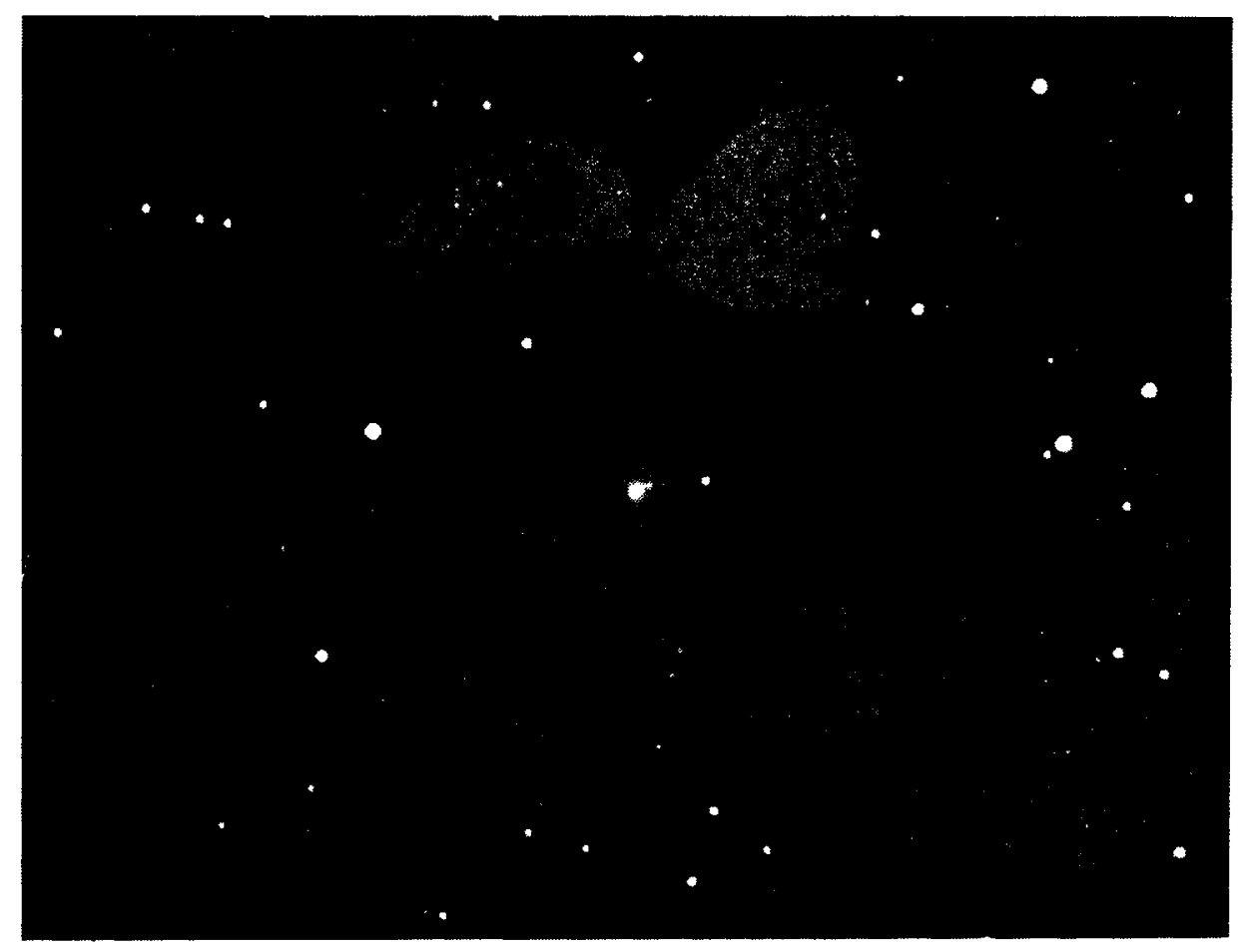

Plate IV. Comet Wirtanen, 1957 VI, 1957 June 27. $\Delta=3.75$ a.u., $\mathbf{r}=4.48$ z.u. 102-cm $\mathrm{f} / 6.8$ reflector, 10-min exposure on Kodak 103a-0 emulsion. (Shows the long-enduring double nucleus.) "Nuclel not quite stellar; $\mathrm{m}_{2}$ 's about 16.3 and 18.0 ; sep. about $8.5 . "$ Official U.S. Navy Photograph 


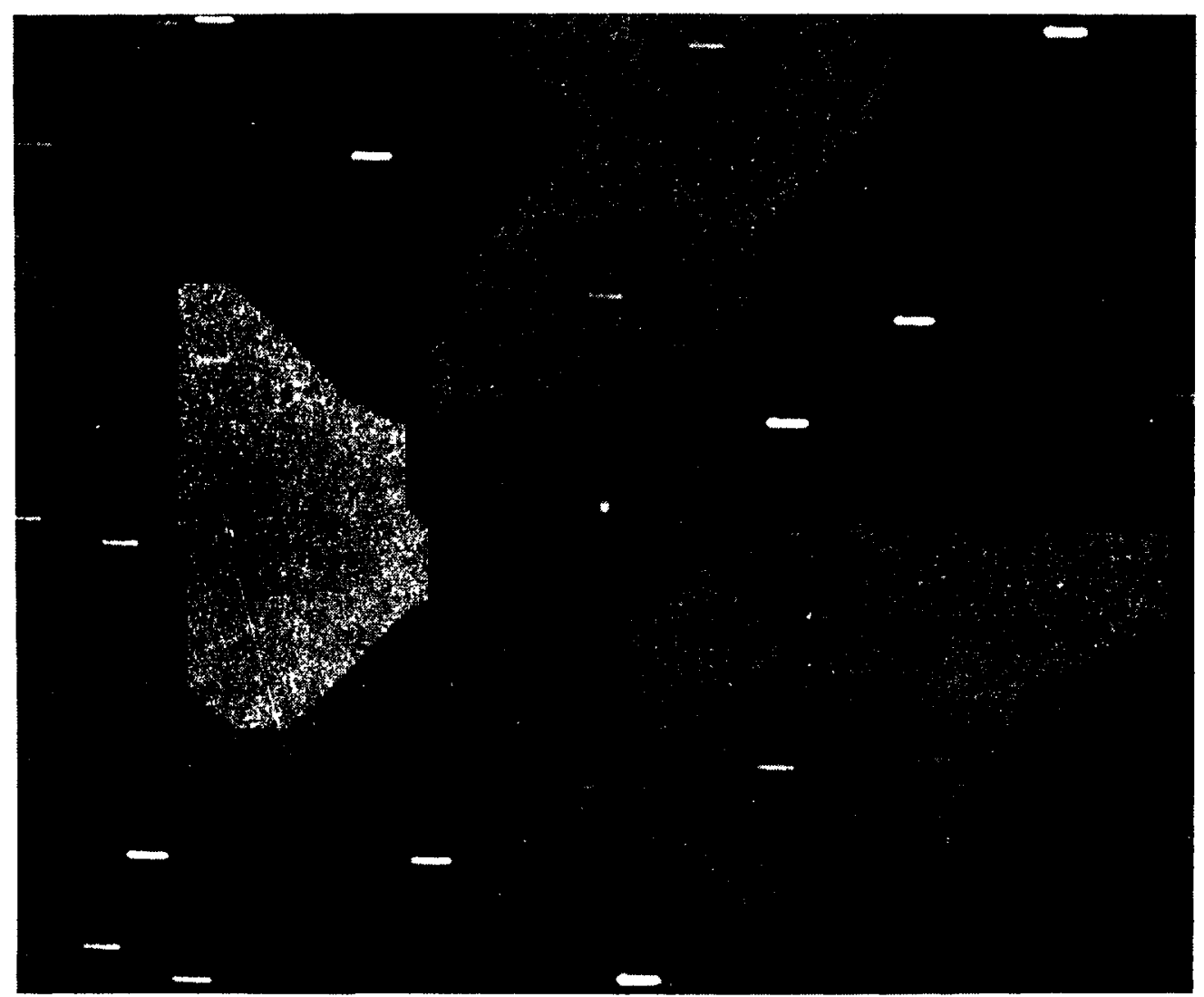

Plate V. Comet Humason, 1962 VIII, 1964 May 12. $\Delta=5.24$ a.u., $\mathbf{r}=5.70$ a u. 102-cm f/6.8 reflector, 120-min exposure on Kodak 103a-0 emulsion. "Practically stellar nuclear condensation of $\mathrm{m}_{2}$ about 17.8 in a weak, almost featureless coma $0: 4$ in diameter." Official U.S. Navy Photograph 


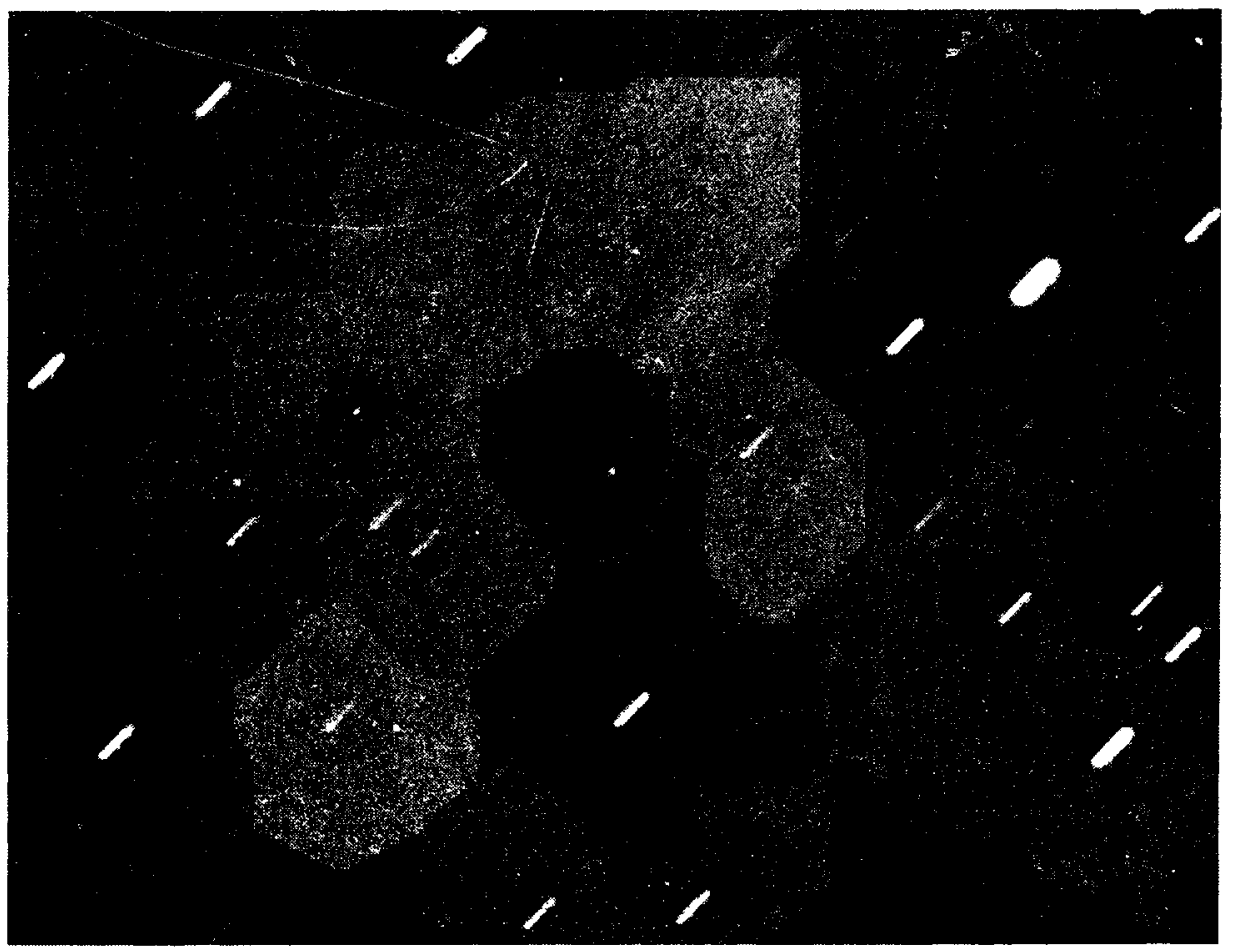

Plate VI. P/Arend-RIgaux, 1957 VII, 1958 May 12. $\Delta=2.04$ a.u., $\mathbf{r}=2.77$ a.u. $102-\mathrm{cm} \mathrm{f} / 6.8$ reflector, $90-\mathrm{mln}$ exposure on Kodak 103a-0 emulsion. "Stellar image of $\mathrm{m}_{2}$ about 19.8." Official U.S. Navy Photograph 


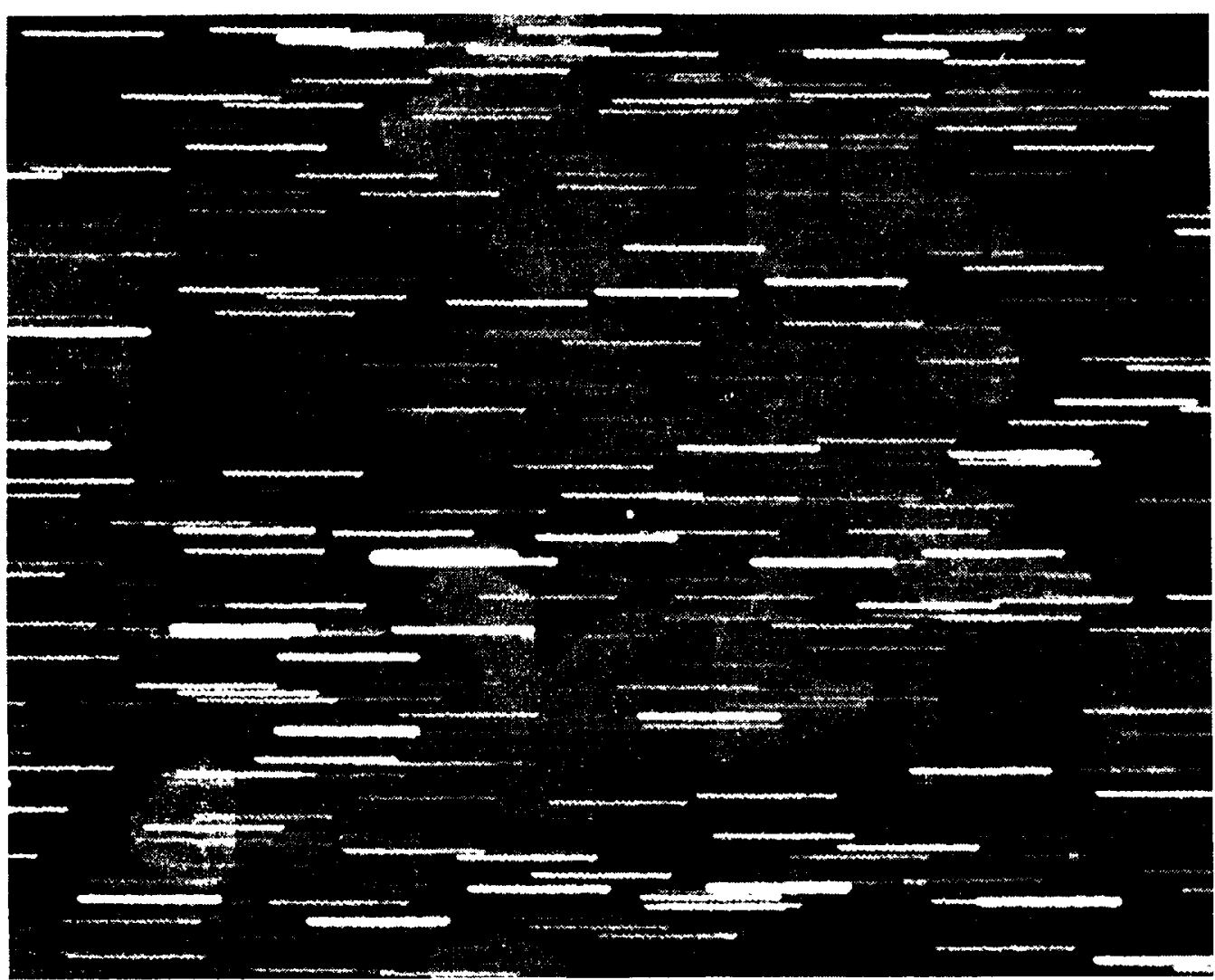

Plate VII. Minor planet (1580) Betulia, 1963 Apr. 25. $\Delta=0.42$ a.u., $r=1.12$ a.u. $102-\mathrm{cm} \mathrm{f} / 6.8$ reflector, 60-min exposure on Kodak 103a-D emision, without filter, prolonged in an effort to record any possible trace of coma that might be present around this close-earth-approach asterold.

"Stellar appearance, magnitude about 16." 
It should be noted that the nuclear dimensions published by the writer were derived from individual magnitude observations, not from absolute magnitudes. The degree of contamination from an unresolved inner coma will vary with time as the intensity of the gas and dust emission fluctuates, and will generally be less at large heliocentric distances. A nuclear absolute magnitude calculated from a collection of observations made over a considerable range in heliocentric distance will include a fit to this contamination. Nuclear radii calculated from absolute magnitudes are likely, therefore, to depend to some degree on the particular range of distances over which the individual objects were observed and the level of their physical activity. Since the overwhelming majority of nuclear magnitude estimates refer to comets at geocentric and heliocentric distances greater than 1 a.u., reduction to unit distance will have the effect of exaggerating the contamination from the come and will lead to spuriously large figures for nuclear dimensions.

Brightness Ephemerides

The apparent brightness of a comet. as it depends on geocentric and heliocentric distance is comonly represented by the relation

$$
I=I_{0} \Delta^{-2} r^{-n}
$$

The nuclear brightness of some comets may show in addition a dependence on phase angle, but such dependence appears to be negligible for total brightness. For ephemeris purposes, the relation (1) is most frequently used in the form

$$
m=m_{0}+5 \log \Delta+2.5 \mathrm{n} \log r
$$

where $\underline{m}$ is now usually specified as referring to "total" magnitude, $m_{1}$, or "nuclear" magnitude, $\mathrm{m}_{2}$. The exponent $\underline{\mathrm{n}}$ thus represents in an average way characteristics of the comet itself and of its response to the solar 
rediation field. The "absolute magnitude", $m_{0}$, corresponds formally to $\Delta=r=1$ a.u., but $1 t$ is not a clear-cut intrinsic property of a comet. For comparison, the conventional form for magnitude ephemerides for minor planets is

$$
m=g+5 \log \Delta+5 \log r+0.023 \alpha^{\circ}
$$

Extensive analyses of the total brightness behavior of observed comets have been made by many investigators (e.g., Bobrovnikoff 194la, 1942, 1943; Schmidt 1951; Vsekhsvyatskij 1958) and have led to identification of several general patterns. "New" comets, defined as those moving in original orbits so nearly parabolic that they are not likely to have passed previously through the inner solar system, are found to be responsive to solar radiation at relatively large distances. Further brightening is comparatively slow on closer approach to the sun. The average value of $\underline{n}$ in (2) is about 3. "Old" comets, including those in definitely elliptical orbits, are more sensitive to decreasing heliocentric distance, and the average value of $\underline{n}$ is found to be larger, nearly 4 for long-period comets, and approaching 6 for short-period comets. Values of $\underline{n}$ found for individual comets in all classes span a wide range, some comets even fading out on approach to perihelion.

For a comet that becomes relatively bright at perihelion but is observed photographically over a long arc, many estimates of $m_{1}$ will be made while the comet is bright, while measures of $\mathfrak{m}_{2}$ will predominate at large $\underline{r}$. Very few comets are observed visually when $m_{1}>12$, while $m_{2}$ observations generally fall in the range $15<\mathrm{m}_{2}<21$. Fits have sometimes been made to the two kinds of magnitudes indiscriminately by adjustment of the parameters in a single formula. When this is done, an exaggerated value of $\underline{n}$ is likely to emerge, along with an $m_{0}$ that is quite uninterpretable. 
When a new comet is discovered visually, the long-focus photographic observer normally expects $m_{2}$ to exceed $m_{1}$ by four to six magnitudes, the amount being sensitive to the diffuseness of the object.

Conversely, it has sometimes been necessary to predict the nearperihelion visual brightness of a comet when only photographic observations at large heliocentric distance are available. This was the situation with Comet Kohoutek in March and April 1973. To arrive at some estimate of the probable development, ephemerides were calculated on the basis of two assumed magnitude laws, one with $\underline{\underline{n}}=4$, and one with $\underline{\mathbf{n}}=6$, each with $\mathrm{m}_{0}$ determined so as to fit the available photographic observations. The more conservative prediction, which turned out to be rather accurate, seems to have been largely overlooked in the excitement that followed. The nearperihelion $m_{1}$ observations of Comet Kohoutek as reported in the IAU Circulars are shown in Fig. 1, with an $\underline{n}=4$ magnitude ephemeris represented by the full curve. A one-magnitude asymmetry in the preperihelion vs. postperihelion brightness behavior is fairly comon, but most comets tend to be somewhat brighter after perihelion passage than before. Although some comets have been followed after perihelion to distances comparable with that at which Comet Kohoutek was discovered, no comet ever before was followed from discovery at a heliocentric distance of nearly 5 a.u. through a perihelion passage less than 0.2 a.u. from the sun. The experience, therefore, was highly instructive.

\section{Summary}

The rate of secular fading of short-period comets continues to be a topic of interest. Visual estimates of total brightness made with small telescopes are the only data comparable with old observations. Interested 


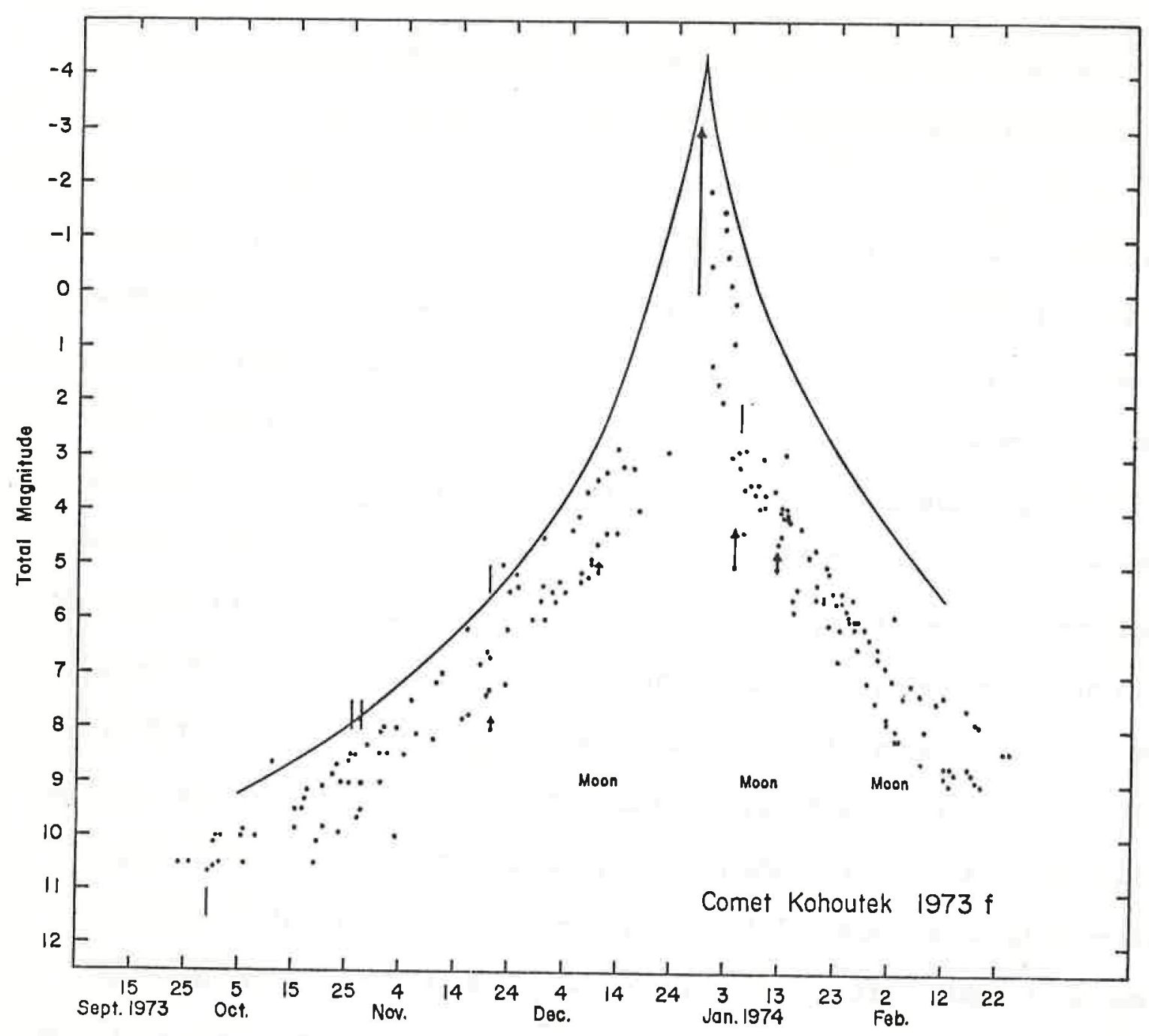

Fig. I

Near-perihelion uncorrected visual estimates of total magnitude $\left(m_{1}\right)$ of Comet Kohoutek, taken from the IAU C1rculars. Observations subject to greater than average uncertainty are represented by vertical lines. Intervals when moonlight may have interfered are marked. The full curve represents a magnitude ephemeris calculated according to an Inverse 4th power dependence on heliocentric distance. 
arateurs have made very useful contributions in this area, and hopefully will continue to do so.

Estimates of nuclear magnitudes seem to have a usefulness beyond that of determining appropriate exposure times for photographic observations, and it appears desirable to encourage activity in this area, particularly since $m_{2}$ 's are an easily obtained by-product of badly needed astrometric observations. Their limitations should be kept in mind, however, when they are used for calculation of dimensions of cometary nuclei. Comparison of results with data obtained by other methods, such as from gas/grain production rates, will give a better idea of the meaning of radii derived from nuclear magnitudes when those are the only data available.

For adequate understanding of the many aspects of physical activity of comets, much more exactly definable data will be needed than have been discussed here. 


\section{ASTROMETRY}

\section{Observations}

Accurately observed right ascensions and declinations as functions of time are the fundamental data for determinations of the orbits of comets. Ephemerides, calculated from orbital elements, are needed even for bright comets if precise physical observations are to be made, and highly accurate predictions of positions in space will be required if spacecraft are to be guided to proper location for in situ experiments. Orbital characteristics of the observed population of comets constitute the basic data for a wide variety of studies of the dynamics of comets, including nongravitational effects, and evolution of orbits.

Precise positions now are determined almost exclusively from measures of photographic plates or films taken either with relatively short-focus, wide-field astrographs or Schmidt cameras, or with large, Iong-focus reflectors of limited field. With short-focus instruments, direct exposures guided at the sidereal rate often are sufficient. The image of the comet on such photographs will be more or less trailed, the amount depending on the total motion relative to stars during the exposure.

With the open scale of the long-focus instruments, or if observations are pushed to the limit with small astrographs, it generally will be advantageous to compensate for the motion of the object during the exposure. The moving object will then appear small and round, while stars are recorded as regular, parallel trails. Basic techniques have been described by Roemer $(1963,1971)$. 
With astrographs and Schmidt cameras, which are guided with an auxiliary telescope, it may be possible to sight directly on the comet, if a sharp condensation can be seen well enough. Or offsetting can be done differentially with respect to a star by use of an eyepiece micrometer, the requirement being that the guide star is brought back after each increment to crosswires systematically displaced by a small amount.

Guiding with large instruments always must be accomplished by offset from a suitable star at the edge of the field being photographed. The direction and rate of motion are calculated in advance from the ephemeris, and the capability must be provided at the telescope for displacement of either the crosswires or the plate by small, accurately definable amounts. It is usually convenient to turn the tailpiece of the telescope so that the displacement is in only one coordinate. Although it is easy in principle to make the offsetting procedure completely automatic by motorizing the guide eyepiece motion and employing an automatic guider, very few telescopes are so equipped at the present.

In extreme situations it may be possible to take adequately compensated plates without corrective guiding if accurately calibrated tracking systems are available in the two telescope coordinates. By careful calculation of the changing effect of refraction on apparent sidereal rate, as well as of the differential motion of the object with respect to stars, satisfactory plates were obtained of Icarus at low altitude in June 1968, completely unguided, when the apparent motion of the minor planet was as great as $24 \mathrm{arcmin} / \mathrm{hr}$. The star trails on the 8-min exposures were $16 \mathrm{~mm}$ long. At the two telescopes used for observations of faint comets at the University of Arizona, the offsetting and guiding aredone by hand. Increments of $0: 5$ are set off at the $229-\mathrm{cm} f / 9$ reflector, and steps of 0.4 
at the $154-\mathrm{cm} \mathrm{f} / 13.5$ reflector at the signal of an automatic timer. Typical intervals between such offsets lie in the range $10-90 \mathrm{sec}$. Most exposures are of duration $10-60 \mathrm{~min}$, but some 90-min exposures have been taken in critical circumstances. The uniformity of the star trails (or the lack of it!) gives valuable information on the smoothness of the tracking and offsetting, and of changes of seeing, transparency, and focus during the exposure. Each of these effects will be recorded quite differently in the star trails and in the image of the faint moving object.

Considerable advantage derives from taking plates in pairs whenever possible, the second observation providing immediate verification of the reality of weak images and a check on the identification of the object through comparison of the observed and computed motions between the exposures.

Photographic Materials

At long $f$ ratios, one generally must use the fastest available photographic emulsions to compress adequate exposures into the time during the night that the faint objects may be in accessible position for observation. At short $\underline{f}$ ratios, enough time may be available to expose fully such efficient but relatively slow plates as hypersensitized Kodak IIIa-J Spectroscopic plates. The best plate for instruments of $\underline{f}$ ratio as long as nine still seems to be the blue-sensitive Kodak 103a-0 emulsion. The quality of images on plates sensitive to a wider wavelength passband is likely to be impaired by atmospheric dispersion, and the use of a filter costs too much light except when observations have to be obtained in difficult special circumstances, such as in bright moonlight. An excellent source for information on available photographic materials, and for guidance on special techniques for hypersensitization, handling, and processing, 
has been compiled by the Eastman Kodak Company (1973). Useful new information on hypersensitization has been published more recently by Scott and Smith (1974) and by Babcock et al. (1974).

Image Tubes and Television-type Sensors

Until recently the field of view obtainable with image tubes was of too limited extent to make such detectors practical for astrometric applications of the kind considered here. Although the cost of the new very large tubes is high, they appear to open the way to some interesting possibilities. A pair of critical observations of the XIIIth satellite of Jupiter were made in September 1974 by R.H. Cromwell, R.J. Weymann, and R. A. McCallister with an image tube having a photocathode nearly $150 \mathrm{~mm}$ In diameter attached to the 229-cm reflector of the Steward Obserratory. Exposures of $90 \mathrm{sec}$ were sufficient to produce adequately exposed images of the satellite, which was of photographic magnitude close to 21.0 at the time of the observations. Two plates were taken to permit identification of the satellite by blink comparison, since the motion was not sufficient during a single exposure to permit recognition of the image of the satellite. Scaled semiaccurate positions suggest that the field characteristics of the ITT F-4094 tube are not a source of gross errors. It would appear that developments in this field of instrumentation, which has been reviewed recently by Livingston (1973), bear close watching.

\section{Measurement}

Whether the motion is compensated, or the comet is allowed to trail, the centers of gravity of the images of comet and reference stars give coordinates referred to the mid-time of the exposure. Measurement may be 
accomplished by direct bisection of the images, if they are small enough, using a suitable coordinate comparator. On long-focus plates it is often necessary to measure the two ends of the star trails, the end coordinates then being averaged to determine those of the center of each star trail. In some cases the trails may be so long, as much as $1-2 \mathrm{~cm}$ in exceptional circumstances, that they cannot be seen in their entirety within the field of view of the optical system of the measuring engine. Obvious problems are likely to arise with measurement of such images.

An impressive variety of measuring machinery has become available within recent years. Some models of coordinate comparators incorporate a considerable degree of automation, but long star trails are beyond the capablity of most of the automatic or semi-automatic machines, at least in their normal mode of operation. Weak images or heavy background fog from moonlight, twilight, or prolonged exposure will pose difficulties with other measuring engines, particularly those in which the viewing is by projection. The comet observer soon becomes accustomed to pursuing his objects under much less than ideal observing conditions, and as a consequence he often acquires material that presents problems during data reduction.

Reduction Techniques

Astrometric reduction methods as applied to determination of positions of comets and minor plonets are fairly well standardized, and a brief review has been given recently by Roemer (1971). Within the field of at least $1^{\circ}$ diameter readily avallable on plates taken with astrographs and Schmidt cameras, it is generally possible to find a suitable configuration of reference stars of reliably known coordinates. A very convenient source for star positions is the Smithsonian Astrophysical Observatory Star 
Catalog (1966). For the Northern Hemisphere, the AGK3, presently available in machine-readable form, offers reference star coordinates and proper motions of even higher precision.

With the limited field of the long-focus instruments ( 30 arcminutes diameter at a scale of $10^{\prime \prime} / \mathrm{mm}$ for the two Arizona telescopes), the only adequate direct source of reference star coordinates is the Astrographic Catalogue. An excellent compendium of detailed information on this, as well as on other standard star catalogs, has been compiled by Eichhorn (1974).

Special problems arise when the Astrographic Catalogue has to be used as the source of reference star positions. For most declination zones in that monumental catalog, only rectangular coordinates measured from photographic plates taken many years ago are directly available for the individual stars. These coordinates can be reduced to right ascension and declination by use of plate constants tabulated in the Catalogue for each field. These preliminary plate constants depend on observations of "repere" stars made as long ago as the $1870^{\circ} \mathrm{s}$ in the Northern Hemisphere, and mostly between 1915 and 1935 in the Southern Hemisphere. These "repère" star positions were used, generally without correction for proper motions, to determine the plate constants referred to the epochs of the plates of the Astrographic Catalogue.

Definitive plate constants are now available for the Northern zones of the Astrographic Catalogue from declination $+90^{\circ}$ to $+32^{\circ}$ (Gllnther and Kox 1970, 1972). And work by P. Lacroute and A.Valbousquet on new constants for the remaining zones of the Northern Hemisphere is in progress at Strasbourg. The new constants are based on the accurate star positions and proper motions that have become available with completion of the AGK3. 
With use of these constants, coordinates for stars of the Astrographic Catalogue are referred consistently to the system of the FK4. New constants for the Northern Hyderobad zone have been published by Eichhorn and Gatewood (1967), and for the Bordeaux zone by Herget (1973) as results of earlier investigations. Improvement in the Southern Hemisphere is not immediately possible, pending further work on the Southern Reference Star progrem.

Somewhat higher accuracy may be obtainable through direct determination of coordinates of secondary reference stars by measurement of a "field" plate. Such a plate is taken with an instrument that records an area large enough to include an adequate set of SAO Catalog stars, and coordinates of selected reference stars within the field of the long-focus instrument are determined from it. This technique of field transfer is a standard one, regularly used, but it requires observations with a second instrument and measurement and reduction of an additional plate. In sparsely populated star fields around the galactic poles, and in the very limited fields that can be photographed with most image tubes, it is the only possible way to obtain accurate positions.

With coordinates of reference stars known from some source catalog, measured coordinates of these stars on the comet plate are used to set up transformation equations between the measured coordinate system and the catalog coordinate system of the reference stars. Then measured coordinates of the comet can be transformed to coordinates in the reference system. Details depend on the nature of the instrument with which the comet has been observed--astrograph or Schmidt camera, Ritchey-Chrétien or classical Cassegrain reflector, with or without correcting optics. A review of classical procedures of both plate constant and dependence types has been given by König (1962). Special formulae applicable to the schmidt have 
been published by Dixon (1962, 1963). Eichhorn (1974) sumarizes information about more sophisticated modern methods.

\section{Accuracy of Positions}

Coordinates of stars in the best reference catalogs may approach or somewhat exceed a systematic accuracy of 0.12 over much of the sky. The accuracy of star positions in the Astrographic Catalogue, referred to the epoch of observation, may approach 0:3. Proper motions, neglected over time spans of more than 70 years in some instances, lead, however, to frequent occurrence of residuals of individual star positions of as much as $1-2^{\prime \prime}$.

Limits on positional accuracy derivable from the comet plate arise from several factors connected to the offsetting of motion, as well as from the comon fact that observations are obtained under less than ideal conditions--at low altitude (refraction, poor seeing), weak images of intrinsicaily very faint objects, or with available exposure time limited by the position of the object in twilight. Large differences in the character of the images of comet and reference stars, which often are mach brighter than the comet and have long, trailed images arising from the differential motion, lead inevitably to a variety of systematic errors. Even if every offset increment is put on regularly and in precisely the right way, and corrective guiding is done accurately, an intrinsic limitation of half the increment size is imposed on precision of the measured position of the comet. Any error in offsetting will lead to incorrect positions of the ends of reference star trails, often without affecting in a noticeable way the image of the comet. Improvement in accuracy is possible by use of stepping motors, by which very small increments can be set off nearly continuously. 
The short exposures possible with image tubes have the advantage that images of both comet and reference stars are nearly round, but the consequence that effects of the frequent large difference in brightness between comet and reference stars are likely to be aggravated. On prolonged exposures during which motion is offset, the images of the reference stars consist effectively of an aligned series of short exposures. Thus image growth from overexposure is lessened, and the detailed structure of trailed Images of even rather bright stars may remain clearly resolvable.

Adequacy of Current Astrometric Programs

The usefulness of observations of position over the longest possible arc for every comet that appears seems to be generally recognized among workers interested in either the physical or dynamical properties of these bodies.

A typical potential observing list in recent years has contained some 12 to 15 objects, at least potentially within the reach of large instruments at any given time. Only three or four of these comets, at most, would be as bright as magnitude 16 or 17 , and thus accessible to very many of the wide-field instruments. Two-thirds or more would be the exclusive responsibility of the observers who use the large, long-focus reflectors. Particularly since the interval of observability amounts to no more than a few months (in some instances, only a few weeks), for some comets, it seems a reasonable goal to try to obtain a few observations of each object each month, in sum total.

Astrometric observations of comets with instruments that reach to magnitude 16-17 are in generally satisfactory state in the Northern Hemisphere, with fairly regular participation of at least 20 observatories 
and of several enthusiastic amateurs who report valuable positions of good accuracy. The situation is less satisfactory in the Southern Hemisphere, even for relatively bright comets. Fairly regular work on comets is included on the programs only at Córdoba, Perth, Wellington, El Leoncito, and at Cerro Tololo. The closing of the Republic Observatory, Johannesburg, has resulted in a serious loss through curtailment of the important observational program on comets and minor planets carried out for many years at the Hartbeespoort Station of that observatory. Northern observers, particularly in Japan, have been very diligent in efforts to obtain observations of objects moving far into the southern skies, so as to lighten the burden that must rest solely on our too few colleagues in the Southem Hemisphere.

The situation for observations of faint comets is more precarious, with regular programs underway only at the University of Arizona, at the Center for Astrophysics (Harvard-Smithsonian), at the Tokyo Observatory, and at Cordoba. Important discoveries and some critical observations come as well from the 122-cm Palomar schmidt telescope. 


\section{REFERENCES}

Babcock, T. A., Sewell, M. H., Lewis, W. C. and James, T. H. (1974)

Hypersensitization of Spectroscopic Films and Plates Using Hydrogen

Gas. Astron. J. 79, 1479.

Beyer, M. (1950) Physische Beobachtungen von Kometen. VII. Astr. Nach. $278,217$.

Bobrownikoff, N. T. (194la) Investigations of the Brightness of Comets. I. Contr. Perkins Obs. No. 15.

- (1941b) Observation of the Brightness of Comets. Popular Astronomy $49,467$.

- (1942) Investigations of the Brightness of Comets. II.

Contr. Perkins Obs. No. 16.

- (1943) On Systematic Errors in the Photometry of Comets. Contr.

Perkins Obs. No. 19.

Delsemme, A. H. and Miller, D. C. (1971) Physico-Chemical Phenomena in

Comets. III. The Continuum of Comet Burnham (1960 II). Planet.

Space Sci. 19, 1229.

Dixon, M. E. (1962, 1963) Astrometry with a Schmidt Camera. M. N. Astr. Soc. South Africa $21,180-186$; $22,6-10,30-36$.

Eastman Kodak Company (1973) Kodak Plates and Films for Sclentific Photography P-315.

Eichhorn, H. and Gatewood, G. D. (1967) Tables Containing the Improved Plate Constants for the Northern Hyderabad Zone of the Astrographic Catalogue. Astron. Contrib. Univ. South Florida at Tampa, No. 3.

Eichhorn, H. (1974) Astronomy of Star Positions New York: Frederick Ungar Gllnther, A. and Kox, H. (1970) Tables of Definitive Plate Constants for the Zones Greenwich, Rome-Vatican, Catania, Helsingfors of the Astrographic Catalogue. Astron. Astrophys. Suppl. Ser. 3, 85. - (1972) Tables of Definitive Plate Constants for the Zones Potsdam, Hyderabad, Uccle, Oxford of the Astrographic Catalogue. Astron. Astrophys. Suppl. Ser. 6, 201.

Herget, $P$ (1973) Plate Constants of the Bordeaux Zone of the Astrographic Catalogue. Cincinnati Obs. Publ. No. 24. Konig, A. (1962) Astrometry with Astrographs In: A. Hiltner, ed., Astronomical Techniques Chicago: Univ. Chicago Press. p. 461 
Livingston, W. C. (1973) Image-Tube Systems. Ann. Rev. Astron. Astrophys. I? 95.

Marsden, B. G. (1974) Comets. Ann. Rev. Astron. Astrophys. 12, 1.

Meisel, D. D. and Morris, C. S. (1975) Comet Brightness Parameters:

Definition, Determination, and Solar Modulation. (Paper 34, this Colloquium)

Morris, C. S. (1973) On Aperture Corrections for Comet Magnitude Estimates. Publ. Astron. Soc. Pacific 85, 470.

Roemer, E. (1961) Astrometric Observations and Orbits of Comets. Astron. J. 66, 368.

- (1963) Comets: Discovery, Orbits, Astrometric Observations.

In: B. M. Middlehurst and G. P. Kuiper, eds., The Moon, Meteorites, and Comets Chicago: Univ. Chicago Press. p. 527.

- (1966) The Dimensions of Cometary Nuclei. Mem. Soc. Roy. des

Sci. de Liège 12, 23.

- (2971) Astrometric Observations. In: T. Gehrels, ed., Physical

Studies of Minor Planets IASA SP-267

Schmidt, M. (1951) The Variation of the Total Brightness of Comets with

Heliocentric Distance. Bull. Astr. Inst. Netherlands $11,253$.

Scott, R. I. and Smith, A. G. (1974) Hypersensitization of Kodak Type 103a-0 Plates by Nitrogen Baking. Astron. J. 72, 656.

Sekanina, Z. (1975) A Continuing Controversy: Has the Cometary Nucleus

Been Resolved? (Paper 38, this Colloquium)

Sidgwick, J. B. (1955) Observational Astronomy for Amateurs Iondon:

Faber and Faber. p. 251.

Smithsonian Astrophysical Observatory (1966) Star Catalog. Publ.

Smithsonian Institution of Washington No. 4562. Washington: Smithsonian Institution.

Vsekhsvyatskij, S. K. (1958) Fizicheskie Kharakteristiki Komet Moscow:

Nauka. (translation 1964 NASA TT F-80) 


\section{DISCUSSION}

B. G. Marsden: I do think that image tubes provide one possible solution to the problem of persuading more observers to do astrometry of faint comets. It is useful to point out that, even though the plates were scaled rather than accurately measured, the Arizona image-tube observations of Jupiter XIII give $(\mathrm{O}-\mathrm{C})$ residuals of no more than about $1.5^{\prime \prime}$.

B. Donn: Regarding nuclear magnitude measurements, there are two possibilities of distinguishing coma contribution from monolithic nuclear magnitude. True nuclear magnitude will be independent of exposure time, whereas a faint coma will show up more on longer exposures. The second, which is more remote, is the measurement of polarization. A dust scattered coma not at opposition will be polarized to some degree, and with a more or less predictable wavelength dependence. What is the observational situation with regard to this?

E. Roemer: I think the second point certainly is well taken, because the fact that you have to keep in mind is that practically everything on my observing list is fainter than 17 th magnitude, and I am hard pressed to get any kind of an image, let alone sort out the ones that might be polarized.

Now, on the occasions where for one reason or another there have been exposures with different exposure times, it is usually a factor of 2 , or something of that sort. On the whole there has been no effect that I can be confident of separating from experimental error-I think there is a 0.2 or 0.3 of a magnitude uncertainty. These are just eyeball estimates; they are comparisons of images with images of stars in the four selected area sequences where there are photoelectric magnitudes down to the 22nd magnitude. Just in compensating for differences in seeing conditions, I do normally put in 0.3 of a magnitude per air mass to account for the differential air mass, but it is kind of a crude comparison. There have been experiments, as Tom Gehrels has gotten rather interested in using an iris photometer on some of the asteroid plates, and, in fact, the quality of those magnitude determinations is poorer than the eyeball, because you simply could not compensate adequately for the difference in the character of the image with the seeing.

Now, there is generally a correlation of these magnitudes with the $\mathrm{f}$-ratio, which is a sign of trouble. The 24-inch at Yerkes and the 82-inch at McDonald that Van Biesbroeck used to use so much are both $\mathrm{f} / 4$, and on the whole Van B's magnitude estimates were the same as those made photographically. I have had some experience with the 24-inch at Yerkes, and I would say estimates are a magnitude brighter than the magnitude estimates that came from the Crossley at $\mathrm{f} / 5.8$ (by Jeffers and some of the people that worked with him). 


\section{DISCUSSION (Continued)}

The Naval Observatory 40 -inch at $\mathrm{f} / 6.8$ I think does lead to magnitudes that are a little bit brighter than the Stewart and Catalina telescopes. I do not get convincing differences between the latter two instruments, at $f / 9$ and $f / 13.5$, so at some stage for a typical sample you do apparently get a long enough $\mathrm{f}$-ratio beyond which you don't get gross differences. However, shorter than, say, f/7 there is enough of a diffuse character to those images where the $\mathrm{f}$-ratio does make a difference much of the time.

P. Wehinger: The 40 and $90 \mathrm{~mm}$ ITT image tubes have resolution well below the seeing disk. The use of such tubes with intermediate band filters is suggested in order to detect fainter comets.

E. Roemer: The trouble is that those fields are too small for us. Our scale in the focal plane on both those telescopes is 10 arc seconds to the millimeter, so it takes us 8 inches to get a 0.5 degree diameter field. We just have to have these huge tubes.

Z. Sekanina: I wonder whether you expect that there are any systematic differences between the "nuclear" magnitudes from your Crossley plates and your 40-inch Ritchey-Chretien plates.

E. Roemer: It is $\mathrm{f} / 5.8$ against $\mathrm{f} / 6.8$. I wouldn't expect a great difference, but it would be in the sense of Crossley brighter. That, by the way, is undoubtedly one of the sources of error in the early estimates of Comet Kohoutek, in that it was Schmidt observations, and then the long focus observations after there already was significant coma development. The Schmidt is running $f / 2.5, \mathrm{f} / 3$, f/3. 5. Those are always going to be less than pure nuclear magnitudes-they are somewhere in between $m_{1}$ 's and $m_{2}$ 's. 